JPP 200355 000-000 (C) 2003 The Authors

Received September 9, 2002 Accepted October 21, 2002 DOI 10.1211/002235702469 ISSN 0022-3573
Medical Mycology Unit, National Institute for Cellular Biotechnology, Department of Biology, National University of Ireland, Maynooth, Co. Kildare, Ireland

Patrick Geraghty, Kevin Kavanagh

Correspondence: K. Kavanagh, Medical Mycology Unit, NICB, Department of Biology, NUI Maynooth, Co. Kildare, Ireland. E-mail: kevin.kavanagh@may.ie

Funding: This work was supported by funding to the National Institute for Cellular Biotechnology under PRTLI 3. Patrick Geraghty is a recipient of a fellowship from the Irish American Partnership.

\section{Erythromycin, an inhibitor of mitoribosomal protein biosynthesis, alters the amphotericin B susceptibility of Candida albicans}

Patrick Geraghty and Kevin Kavanagh

\begin{abstract}
Exposure of the yeast Candida albicans to the macrolide antibiotic erythromycin $\left(\mathrm{C}_{37} \mathrm{H}_{67} \mathrm{NO}_{13}\right)$ results in elevated tolerance to the polyene antifungal amphotericin B. Erythromycin displays no fungistatic activity against $C$. albicans but inhibits the synthesis of cytochromes, particularly cytochrome aa $a_{3}$. Consequently there is a reduction in aerobic respiration by up to $90 \%$ when cells are exposed to $10 \mathrm{mg} \mathrm{mL}^{-1}$ erythromycin. Cellular ergosterol levels are also severely reduced. Erythromycin inhibits protein biosynthesis in ribosomes (mitoribosomes) located within the mitochondrion of the yeast cell, which results in a disruption of cytochrome biosynthesis with an adverse effect on respiration. The synthesis of ergosterol is oxygen dependent and consequently ergosterol levels are depleted in erythromycin-treated C. albicans. Ergosterol is the target for amphotericin B and since there is less of this sterol in erythromycin-treated cells, there is an increase in tolerance of the antifungal agent. Our work indicates that co-administration of erythromycin and amphotericin B to control bacterial and fungal infections, respectively, may inadvertently lead to an elevation in the tolerance of C. albicans for this antifungal agent.
\end{abstract}

\section{Introduction}

The yeast Candida albicans is an opportunistic fungal pathogen that is capable of inducing a range of superficial and systemic infections in the immunocompromised host (Odds 1996). The incidence of infections due to yeast of the genus Candida has increased dramatically in the last two decades due, in part, to the advent of diseases such as AIDS (Gilfillan et al 1998), the use of immunosuppressive therapy as a prerequisite to organ transplantation (Lunel et al 1999) and the increasing number of immunocompromised patients (Pfaller et al 1998).

Conventional therapy for the control of infection by $C$. albicans relies upon the use of a range of polyene and azole antifungal agents (Munoz et al 2000) that bind the fungal membrane sterol ergosterol (Abu-Salah 1996) or disrupt sterol biosynthesis, respectively. In the case of polyene drugs, the binding of ergosterol leads to the formation of pores in the fungal cell membrane, metabolite leakage and subsequent cell death (Abu Salah 1996). Azoles disrupt ergosterol synthesis and induce the formation of toxic sterol intermediates, which prove lethal to the cell (White et al 1998). The polyene antifungal agent amphotericin B is considered to be the gold standard of antifungal chemotherapy and is generally reserved for life-threatening systemic infections due to its extreme nephrotoxicity (Polak 1999). Antifungal therapy can be compromised by the appearance of isolates that may be inherently resistant to specific antifungals or develop resistance after prolonged exposure. The main mechanisms conferring resistance to azole drugs include increased expression of efflux pumps, altered target sites and modifications of steps leading to ergosterol biosynthesis (White et al 1998). Resistance or tolerance to polyene drugs is clinically rare but can be mediated by reductions in the sterol content of the fungal cell membrane (Kelly et al 1997).

Fungal infections in immunocompromised patients are frequently found in association with bacteraemia, which creates the possibility that drugs used to control bacterial infections can have an adverse impact on the therapeutic outcome of a fungal infection. Erythromycin $\left(\mathrm{C}_{37} \mathrm{H}_{67} \mathrm{NO}_{13}\right)$ is a macrolide antibiotic originally isolated from Streptomyces erytheus, with activity against Gram-positive and Gram-negative bacteria. 
It contains a large (14-carbon) lactone ring, which may be substituted with one or more sugar residues. Erythromycin functions by binding the $50 \mathrm{~S}$ ribosomal sub-unit and inhibiting translocation of the elongated peptide from one binding site to another on the ribosome thus inhibiting the addition of amino acids to the growing peptide chain (Ibrahim \& Beattie 1973; Gale et al 1981). Erythromycin therapy leads to a modest increase in the $C$. albicans population of the upper gastro-intestinal tract of mice (Samonis et al 2002).

The mitochondrion is the site of phosphorylation of ADP to ATP and is the site of aerobic respiration within the fungal cell. Fungal mitochondria are semi-autonomous organelles within the cell and contain ribosomes (referred to as mitoribosomes), which are prokaryotic in nature and sensitive to antibiotics (eg. chloramphenicol, erythromycin) unlike their cytoplasmic counterparts (Bottger et al 2001). Mitoribosomes are distinguishable from cytoplasmic ribosomes based on sedimentation coefficients, with the latter ribosomes being $80 \mathrm{~S}$ and composed of $60 \mathrm{~S}$ and $40 \mathrm{~S}$ sub-units while yeast mitoribosomes are approximately 74 S with $50 \mathrm{~S}$ and $37 \mathrm{~S}$ sub-units (Whittaker \& Danks 1978). Antibiotics that affect bacteria also affect fungal mitoribosmes, inhibiting the activity of the large 50S sub-units (Bottger et al 2001). Recent work has demonstrated that erythromycin retards the ageing process in Saccharomyces cerevisiae by interfering with mitoribosome function (Holbrook \& Menninger 2002); consequently, there is the possibility that erythromycin therapy to treat a bacterial infection could result in an alteration in the activity of fungal mitoribosomes with a deleterious effect on the outcome of fungal therapy.

The aim of the work presented here was to establish the nature of the interaction of erythromycin with $C$. albicans and to determine whether this has any role in moderating the susceptibility of $C$. albicans to antifungal therapy.

\section{Materials and Methods}

\section{Yeast isolates and culture conditions}

Candida albicans MEN (serotype B, originally isolated from an eye infection and a gift from Dr D. Kerridge, Cambridge, UK) was used throughout this study as it is a clinical isolate with a well characterised response to antifungal agents. Cultures were grown in YEPD (yeast extract-peptone-Dglucose) broth $(2 \% \mathrm{w} / \mathrm{v}$ glucose (Sigma-Aldrich Chemical Co. Ltd, Dorset, UK), 2\% w/v bactopeptone (Difco Laboratories, MI) and $1 \% \mathrm{w} / \mathrm{v}$ yeast extract (Oxoid, Basingstoke, UK)) to the stationary phase. Stationaryphase cultures had a typical cell viability of $77.4 \pm 6.2 \%$. Where appropriate, media were solidified by the addition of $2 \% \mathrm{w} / \mathrm{v}$ agar (Oxoid). Yeast cultures were maintained on YEPD agar at $4{ }^{\circ} \mathrm{C}$ and sub-cultured every 4-6 weeks.

\section{Erythromycin toxicity assay}

Erythromycin (Sigma Aldrich) was dissolved at a concentration of $500 \mathrm{mg} \mathrm{mL}^{-1}$ in dimethyl sulfoxide (DMSO).
This was diluted with growth medium to give the appropriate working dilutions. Yeast cultures were grown to the stationary phase (approximately $1.5 \times 10^{8} \mathrm{~mL}^{-1}$ ) in YEPD overnight at $30^{\circ} \mathrm{C}(200$ rotations per minute on an orbital shaker). The cells were counted and $5 \times 10^{6}$ cells were added to $10 \mathrm{~mL}$ of YEPD broth containing erythromycin (Sigma-Aldrich) at 2.5, 5 or $10 \mathrm{mg} \mathrm{mL}^{-1}$. A DMSO control was included. The cell suspensions were incubated at $30^{\circ} \mathrm{C}$ for $24 \mathrm{~h}$, after which time the final cell density was established microscopically.

\section{Antifungal susceptibility testing}

Yeast cultures were grown to the stationary phase in antibiotic medium 3 (AB 3) (Oxoid) overnight at $30^{\circ} \mathrm{C}$ and 200 rotations per minute, harvested by centrifugation ( $2220 \mathrm{~g}$ for $5 \mathrm{~min}$ in a Beckmann GS-6 centrifuge) and diluted to $1 \times 10^{6}$ cells $/ \mathrm{mL}$ in AB3. Cells $\left(1 \times 10^{5}\right.$ in $100 \mu \mathrm{L})$ were added to each well of a 96-well plate containing amphotericin B (Sigma-Aldrich) dissolved in AB3 in serial dilutions from 1.25 to $0.0025 \mu \mathrm{g} \mathrm{mL}^{-1}$. The plates were incubated at $30^{\circ} \mathrm{C}$ for $24 \mathrm{~h}$ and the optical density was read at $540 \mathrm{~nm}$ using a microplate reader (Labs Systems iEMS). The MIC80 was determined to be the lowest concentration of amphotericin $\mathrm{B}$ required to reduce growth by $80 \%$ relative to the control (Moran et al 1997).

\section{Measurement of oxygen uptake}

Stationary-phase cells (approximately $1.5 \times 10^{8}$ cells $/ \mathrm{mL}$ ) grown in YEPD broth at $30^{\circ} \mathrm{C}$ overnight were harvested, washed with phosphate-buffered saline (PBS) and resuspended in $0.025 \mathrm{M}$ phosphate buffer $(\mathrm{pH} 7.2)$ at a density of $5 \times 10^{8} \mathrm{~mL}^{-1}$. Oxygen uptake measurements were made at $30^{\circ} \mathrm{C}$, using a Clark-type oxygen electrode. Oxygen uptake rates were calculated as $\mu \mathrm{mol}$ of oxygen consumed in $30 \mathrm{~s}$ per $10^{8}$ cells.

\section{Cytochrome analysis}

Stationary-phase cells $\left(4 \times 10^{9}\right.$ in total) were harvested by centrifugation and washed twice in PBS ( $\mathrm{pH} 7.2)$. Half of the sample was oxidised by suspending in $20 \mathrm{~mL}$ of $0.3 \%$ $\mathrm{w} / \mathrm{v}$ sodium hypochlorite (Sigma-Aldrich). The cells were harvested and resuspended in $50 \% \mathrm{v} / \mathrm{v}$ glycerol. The remaining $2 \times 10^{9}$ cells were resuspended in $50 \% \mathrm{v} / \mathrm{v}$ glycerol to which a few crystals of sodium dithionite (Merck, Darmstadt, Germany) were added to reduce the cytochromes. Reduced-oxidised differential spectra were measured on a dual beam Cary IE UV - VISIBLE spectrophotometer over $500-650 \mathrm{~nm}$.

\section{Sterol extraction and analysis}

Sterols were extracted using the method of ArthingtonSkaggs et al (1999) with slight modifications. Stationaryphase cells ( $1 \mathrm{~g}$ wet weight) were harvested and washed with PBS. Cells were resuspended in $20 \% \mathrm{w} / \mathrm{v} \mathrm{KOH}$ and $60 \% \mathrm{v} / \mathrm{v}$ ethanol and placed in a shaking water bath $(80$ $90{ }^{\circ} \mathrm{C}$ ) for $90 \mathrm{~min}$. N-heptane was added to the solution, 
which was vigorously agitated for $10 \mathrm{~s}$ and the aqueous (upper) layer was removed. The sterol content of the hexane layer was quantified using a dual beam spectrophotometer over the range $240-320 \mathrm{~nm}$. An ergosterol standard curve was calculated over the range $100-0.25 \mu \mathrm{g} \mathrm{mL}^{-1}$ and the range of linearity was $0.25-6.25 \mu \mathrm{g} \mathrm{mL}^{-1}$. The lowest level of detection of ergosterol was $0.1 \mu \mathrm{g} \mathrm{mL}^{-1}$.

\section{Statistical analysis}

The Kruskal-Wallis test was performed on the results recorded from the amphotericin B susceptibility tests, cell respiration and sterol determinations with a statistical package (SigmaStat Statistical Analysis System, Version 1.00). All sets of results displayed a significant difference with respect to the controls. In the case of respiration there is a statically significant difference $(P=0.03)$, while in the amphotericin B susceptibility determinations there is a statically significant difference $(P<0.04)$. In the sterol determinations the difference between the control and the treatments is significant at $P<0.001$.

\section{Results}

\section{Effect of erythromycin on the growth of C. albicans}

Erythromycin displays activity against Gram-positive and Gram-negative bacteria by interfering with the functioning of bacterial ribosomes which are structurally similar to eukaryotic mitoribosomes (Bottger et al 2001). As a consequence, it is possible that erythromycin may display fungistatic activity against $C$. albicans. YEPD broth supplemented with $2.5,5$ or $10 \mathrm{mg} \mathrm{mL}^{-1}$ erythromycin was inoculated with $C$. albicans $\mathrm{MEN}$ at an initial cell density of $5 \times 10^{5} \mathrm{~mL}^{-1}$. Cultures were incubated for $24 \mathrm{~h}$ at $30^{\circ} \mathrm{C}$ and the final cell density was determined by haemocytometer count. The results indicated that at concentrations of $2.5-10 \mathrm{mg} \mathrm{mL}^{-1}$ erythromycin displayed no substantial fungistatic effect on $C$. albicans (data not presented).

\section{Effect of erythromycin on susceptibility of C. albicans to amphotericin B}

The simultaneous occurrence of bacterial and fungal infections in immunocompromised patients creates the possibility of antibacterial and antifungal drugs interacting with each other, or the target organism of the other agent, thus compromising therapy. Amphotericin B is a polyene antifungal originally isolated from Streptomyces nodosus and used in the treatment of life-threatening systemic mycoses (Polak 1999). It functions by binding ergosterol in the fungal cell membrane and creating a pore, which leads to loss of metabolites and acidification of the cytoplasm (Abu-Salah 1996). Toxicity assays were performed to establish whether cells pre-treated with erythromycin had altered susceptibility to amphotericin B. Cells pre-exposed to erythromycin displayed amphotericin B MIC80 values increased by approximately $80-110 \%$ (Figure 1). In the case of control cells the MIC80 value was $0.018 \mu \mathrm{g} \mathrm{mL}^{-1}$ whereas in cells pre-grown in the presence of $2.5,5$ or $10 \mathrm{mg} \mathrm{mL}^{-1}$ erythromycin the MIC80 ranged from 0.033 to $0.038 \mu \mathrm{g} \mathrm{mL}^{-1}$. Comparable toxicity assays were performed using the azole drugs clotrimazole, ketoconazole and miconazole and no alteration in antifungal susceptibility was detected following pre-growth of C. albicans in erythromycin.

\section{Effect of erythromycin on cellular respiration of C. albicans}

Erythromycin inhibits the action of mitoribosomes by preventing protein elongation at the transpeptidation step (Gale et al 1981) — a process that could disrupt mitochondrial respiration since a number of the subunits of the cytochromes involved in the electron transfer pathway of respiration are synthesised on mitoribosomes (Whittaker \& Danks 1978). The cytochrome profiles of cells treated with erythromycin were obtained to establish the effect of this drug on cytochrome biosynthesis. The cytochrome profile (Figure 2) of control cells indicated the presence of cytochromes $\mathrm{aa}_{3}(602 \mathrm{~nm}), \mathrm{b}(564 \mathrm{~nm})$ and c $(550-554 \mathrm{~nm})$, but these were altered in those cells exposed to erythromycin. In particular, the cytochrome $\mathrm{aa}_{3}$ peak

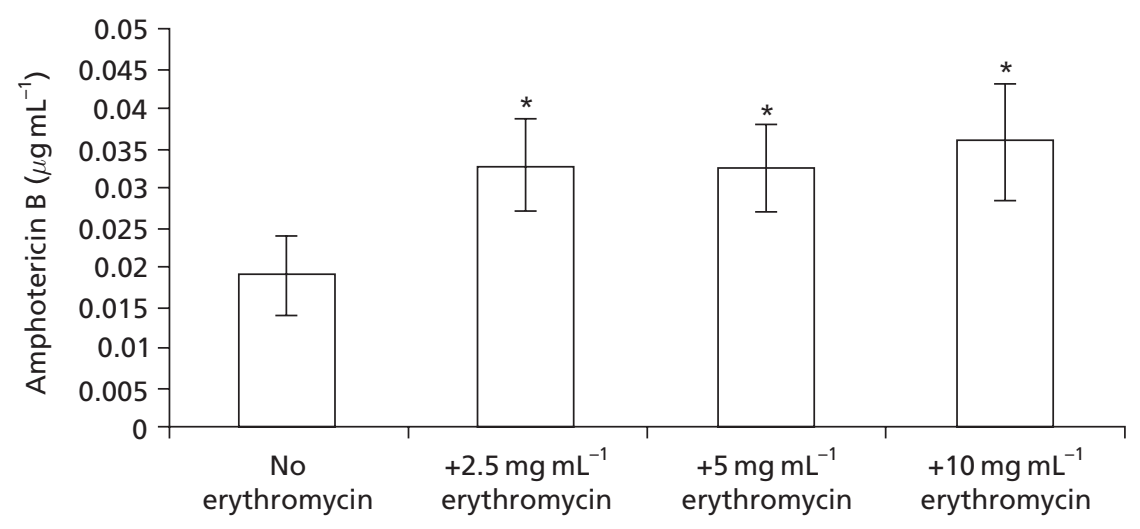

Figure 1 Susceptibility of Candida albicans to amphotericin B following growth in erythromycin, expressed as MIC80. Data are means \pm s.e.m. of 5 independent determinations; ${ }^{*} P<0.04$ vs no erythromycin. 


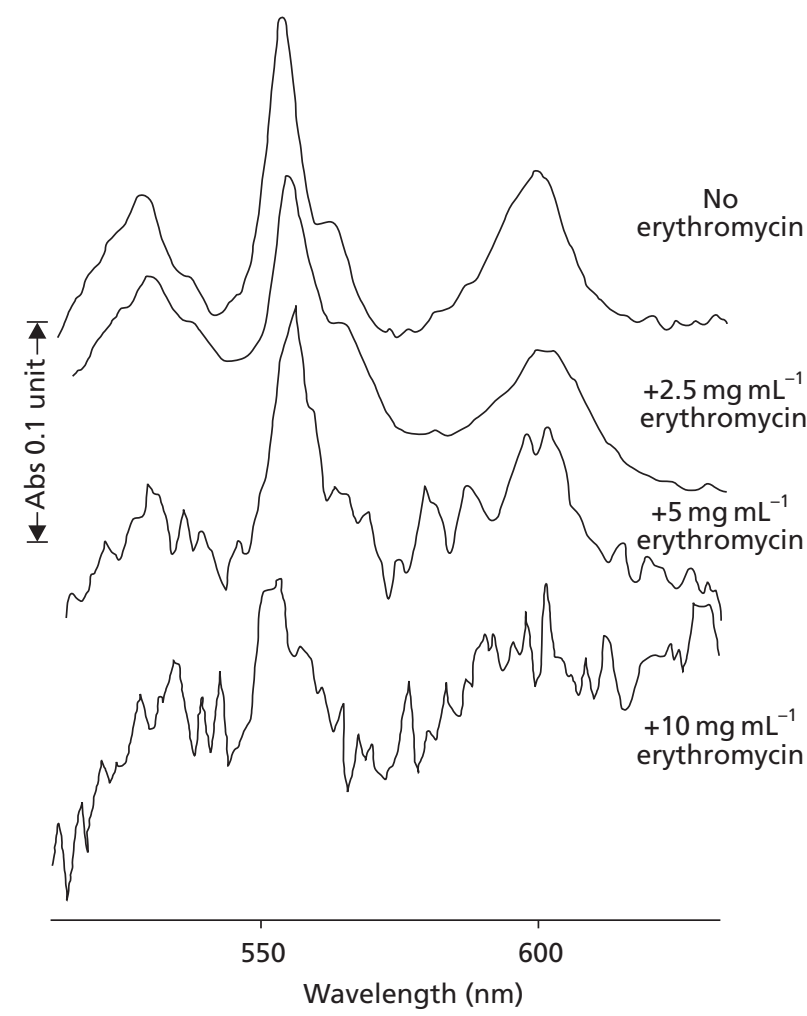

Figure 2 Differential spectra of mitochondrial cytochromes of Candida albicans following growth in erythromycin. Cytochrome $\mathrm{aa}_{3}(602 \mathrm{~nm})$, cytochrome b $(564 \mathrm{~nm})$ and cytochrome c (550$554 \mathrm{~nm})$.

was severely disrupted in the cultures treated with 5 and $10 \mathrm{mg} \mathrm{mL}^{-1}$ erythromycin.

The disruption of cytochrome biosynthesis following growth in erythromycin has the potential to adversely affect cellular respiration, since an incomplete electron transfer pathway would result in the cells. The respiration rates of cultures grown in erythromycin was determined using a Clark-type oxygen electrode. The results indicate that exposure to $2.5,5$ or $10 \mathrm{mg} \mathrm{mL}^{-1}$ erythromycin depresses oxygen consumption (Figure 3) - in the case of cells treated with $10 \mathrm{~m} \mathrm{~mL}^{-1}$ erythromycin by up to $90 \%$.

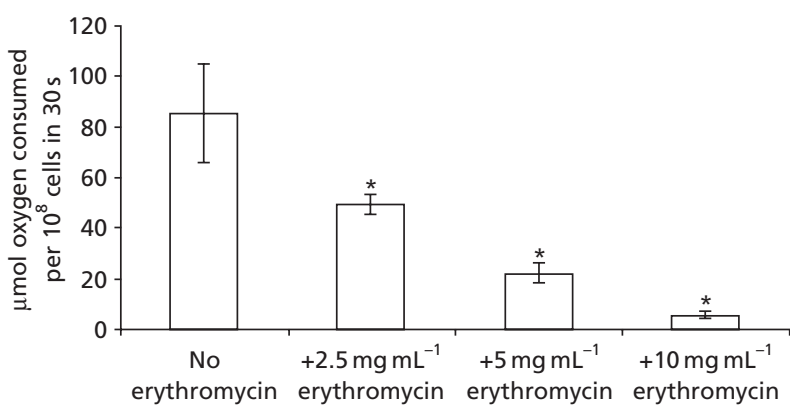

Figure 3 Effect of erythromycin on respiration of Candida albicans. Data are means \pm s.e.m. of 5 independent determinations; ${ }^{*} P=0.03$ vs no erythromycin.

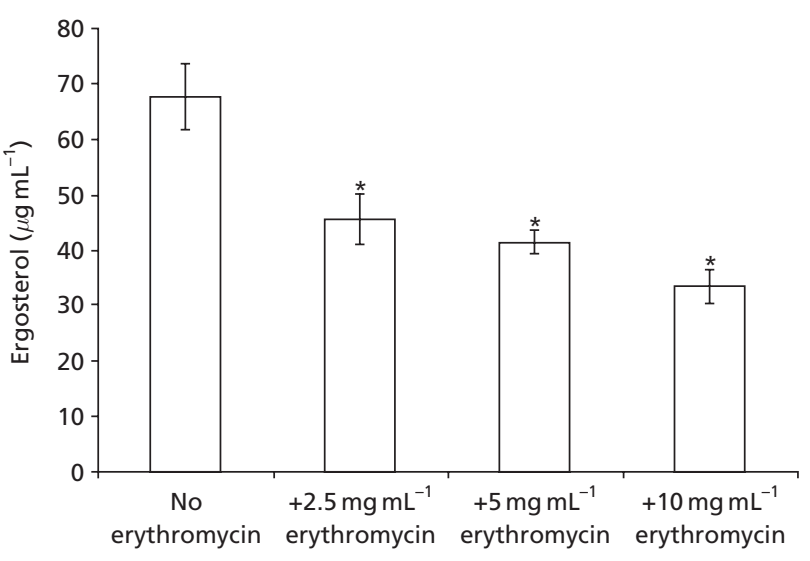

Figure 4 Effect of erythromycin on ergosterol content of Candida albicans. Data are means \pm s.e.m. of 5 independent determinations; ${ }^{*} P<0.0001$ vs no erythromycin.

\section{Effect of erythromycin on ergosterol content of C. albicans}

Ergosterol is a key component of the fungal cell membrane and the target for amphotericin B (Abu Salah 1996). The conventional belief is that amphotericin B functions by binding ergosterol and forming a pore in the cell membrane, which leads to leakage of metabolites and acidification of the cytoplasm. Resistance to amphotericin B is rare but where it has been recorded there has been a reduced level of ergosterol in the cell membrane, providing fewer binding sites for the polyene antifungal (Kelly et al 1997). The ergosterol content of cells treated with erythromycin was determined to establish whether a reduction in ergosterol content could be a mechanism for the increased tolerance to amphotericin B. Ergosterol was extracted from control cells and cells grown in the presence of 2.5, 5 and $10 \mathrm{mg} \mathrm{mL}^{-1}$ erythromycin, and quantified by scanning over the range $240-330 \mathrm{~nm}$ in a dual beam spectrophotometer. Ergosterol produces a characteristic 4-peak profile in the range $240-300 \mathrm{~nm}$ and this technique can be used to quantify levels within fungal cells (Arthington-Skaggs et al 1999). The results (Figure 4) indicate a reduction in ergosterol content for the three erythromycintreated cultures, with the greatest reduction being evident in cultures treated with $10 \mathrm{mg} \mathrm{mL}^{-1}$ erythromycin. Cells grown in the presence of $2.5 \mathrm{mg} \mathrm{mL}^{-1}$ erythromycin had an ergosterol level approximately $66 \%$ of the control while those cells grown in $10 \mathrm{mg} \mathrm{mL}^{-1}$ had an ergosterol level half the control level.

\section{Discussion}

The results presented in this paper indicate that erythromycin has no significant effect on the growth of C. albicans but that pre-growth of cells in the presence of erythromycin alters the susceptibility of cells to amphotericin B (Figure 1). Erythromycin acts on mitochondrial protein biosynthesis, which leads to a reduction in the 
amount of cytochrome $\mathrm{aa}_{3}$ (Figure 2). In addition, there is some disruption of cytochromes $b$ and $c 1$. Three of the seven sub-units of cytochrome $\mathrm{aa}_{3}$ are mitochondrially encoded, being synthesised on mitoribosomes (Whittaker \& Danks 1978). Cytochrome $\mathrm{aa}_{3}$ is an integral part of the electron transfer pathway and its suppression could explain the reduction in oxygen consumption evident in erythromycin-treated cells.

Treatment of $C$. albicans with erythromycin gives a substantial reduction in respiration, with oxygen uptake being reduced by up to $90 \%$ when cells were grown in $10 \mathrm{mg} \mathrm{mL}^{-1}$ erythromycin (Figure 3). Cells exposed to erythromycin also show a reduction in the content of ergosterol (Figure 4), which is the target of amphotericin B. Fungal cells require oxygen to synthesise ergosterol and a reduction in respiratory efficiency or an inability to respire leads to reduced levels of this important membrane sterol. Reduction in sterol levels in C. albicans has been identified previously as a mechanism for increased growth in the presence of amphotericin B (Kelly et al 1997; White et al 1998). Disruption of the genes in the ergosterol biosynthesis pathway gives decreased ergosterol in Candida glabrata and an increase in drug tolerance, particularly to amphotericin B (Geber et al 1995; Vazquez et al 1996). The requirement for a functional mitochondrion in ergosterol biosynthesis is well characterised and arises from the provision of NADPH for squalene dimerisation (Parks \& Casey 1995). In addition, Ergl encodes squalene epoxidase which converts squalene to 2,3-oxidosqualene, which is an oxygen-dependent step and in a cell with reduced respiration there would be little synthesis of ergosterol (Daum et al 1998) thus leading to the reduced ergosterol content evident in erythromycin-treated cells.

The work presented here, demonstrates that growth of C. albicans in the presence of erythromycin suppresses the synthesis of mitochondrial cytochromes, particularly cytochrome $\mathrm{aa}_{3}$, which in turn leads to a reduction in cellular respiration. Reduced oxygen uptake leads to a reduction in the amount of ergosterol in the fungal cell membrane due to the requirement for oxygen in sterol biosynthesis. Reduced ergosterol levels have previously been shown to give an elevated tolerance of amphotericin B in C. albicans (Kelly et al 1997).

The data presented here suggest that co-administration of erythromycin and amphotericin B to treat bacterial and fungal infections, respectively, may lead to an elevation in the tolerance of $C$. albicans for the polyene antifungal, with potentially deleterious consequences for patient recovery. The use of amphotericin B is reserved for severe systemic fungal infections and, as such, represents the last line of defence in severely immunocompromised patients (Polak 1999). Clinical resistance to this polyene is rare (White et al 1998). However, our work indicates that a drug that reduces the ergosterol content of the fungal cell membrane will result in an elevation in the tolerance of amphotericin B. This may be sufficient to reduce the efficacy of antifungal therapy and lead to increased patient mortality. Erythromycin treatment had no effect on the tolerance of $C$. albicans for azole drugs, which is not unexpected since azoles target steps in the biosynthesis of ergosterol rather than the molecule itself and consequently their action is not dependent upon the presence of ergosterol in the fungal cell membrane.

In terms of patient care, caution should be exercised in the administration of drugs for the control of bacterial infections that may inhibit fungal respiration since this leads to a reduction in ergosterol and a concomitant elevation in tolerance of amphotericin B. This phenomenon may be clinically important where there is prolonged therapy with erythromycin (or other antibiotics that interfere with fungal respiration) and amphotericin B since such conditions may increase the proportion of the fungal population exhibiting elevated tolerance to the latter agent.

In conclusion, this work demonstrates that erythromycin inhibits respiration and mitochondrially encoded cytochrome biosynthesis in $C$. albicans, which leads to a reduction in ergosterol levels in the fungal cell membrane and an increased tolerance to the antifungal agent amphotericin B.

\section{References}

Abu-Salah, K. M. (1996) Amphotericin B: an update. Br. J. Biomed. Sci. 53: 122-133

Arthington-Skaggs, B. A., Warnock, D. W., Morrison, C. J. (1999) Quantitation of ergosterol content: novel methods for determination of fluconazole susceptibility of Candida albicans. J. Clin. Microbiol. 37: 3332-3337

Bottger, E. C., Springer, B., Prammananan, T., Kidan, Y., Sander, P. (2001) Structural basis for selectivity and toxicity of ribosomal antibiotics. EMBO Rep. 21: 318-323

Daum, G., Lees, N. D., Bard, M., Dickson, R. (1998) Biochemistry, cell biology and molecular biology of lipids of Saccharomyces cerevisiae. Yeast 14: 1471-1510

Gale E. F., Cundliffe, E., Reynolds, P. E., Richmond, M. H., Waring, M. J. (1981) The molecular basis of antibiotic action. 2nd Edition, John Wiley \& Sons, London

Geber, A., Hitchcock, C. A., Swartz, J. E., Pullen, F. S., Marsden, K. E., Kwon-Chung, K. J., Bennett, J. E. (1995) Deletion of the Candida glabrata ERG3 and ERG11 genes: effect on cell viability, cell growth, sterol composition and antifungal susceptibility. Antimicrob. Agents Chemother. 39: $2708-2717$

Gilfillan, G. D., Sullivan, D. J., Haynes, K., Parkinson, T., Coleman, D. C., Gow, N. A. R. (1998) Candida dubliniensis: phylogeny and putative virulence factors. Microbiology 144: 829-838

Holbrook, Menninger, J. R. (2002) Erythromycin slows ageing of Saccharomyces cerevisiae. J. Gerontol. 57: 29-36

Ibrahim N. G., Beattie, D. S. (1973) Protein synthesis on ribosomes isolated from rat liver mitochondria: sensitivity to erythromycin. FEBS Lett. 36: 102-104

Kelly, S. I., Lamb, D. C., Kelly, D. E., Manning, N. J., Loeffier, J., Herbart, H., Schumacher, U., Einsele, H. (1997) Resistance to fluconazole and cross resistance to amphotericin B in Candida albicans from AIDS patients caused by defective sterol $\Delta^{5,6}$ desaturation. FEBS Lett. 400: 80-82

Lunel, F. M. V, Meis, F. G., Voss, A. (1999) Nosocomial fungal infections: candidemia. Diagn. Microbiol. Infect. Dis. 34: 213-220

Moran, G., Sullivan, D. J., Henman, M. C., McCreary, C. E., Harrington, B. J., Shanley, D. B., Coleman, D. C. (1997) Antifungal drug susceptibilities of oral Candida dubliniensis isolates 
from human immuno-deficiency virus (HIV)-infected and nonHIV-infected subjects and generation of stable fluconazole resistant derivatives in vitro. Antimicrob. Agents Chemother. 41: 617-623

Munoz, P., Burilo, A., Bouza, E. (2000) Criteria used when initiating antifungal therapy against Candida spp. in the intensive care unit. Int. J. Antimicrob. Agents 15: 83-90

Odds, F. C. (1996) Epidemiological shifts in opportunistic and nosocomial Candida infections: mycological aspects. Int. J. Antimicrob. Agents 6: 141-144

Parks, L. W., Casey, W. M. (1995) Physiological implications of sterol biosynthesis in yeast. Ann. Rev. Microbiol. 49: 95-116

Polak, A. (1999) The past, present and future of antimycotic combination therapy. Mycoses 42: 355-370

Pfaller, M. A., Jones, R. N., Messer, S. A. Edmond, M. B., Wenzel, R. P. (1998) National surveillance of nosocomial blood stream infection due to Candida albicans: frequency of occurrence and anti-fungal susceptibility in the SCOPE programme. Diagn. Microbial Infect. Dis. 31: 327-332

Samonis, G., Maraki, S., Anatoliotakis, N., Anatoliotaki, M., Apostolakou, H., Margioris, A., Tselentis, Y., Kontoyiannis, D. (2002) Effects of erythromycin, clarithromycin, roxithromycin and azithromycin on murine gut colonisation by Candida albicans. Med. Mycol. 40: 139-142

Vazquez, J. A., Arganoza, M. T. Vaishampayan, J. K., Akins, R. A. (1996) In vitro interaction between Amphotercin B and azoles in Candida albicans. Antimicrob. Agents Chemother. 40: 25112516

White, T. C., Marr, K. A., Bowden, R. A. (1998) Clinical, cellular and molecular factors that contribute to anti-fungal drug resistance. Clin Microbiol. Rev. 11: 382-402

Whittaker, P. A., Danks, S. M. (1978) Mitochondria: structure, function and assembly. Integrated themes in biology. Longman, London 